\title{
A rare cause of a right atrial mass
}

\author{
Ho-fon Royce Law ${ }^{1 *}$, Rory Beattie ${ }^{2}$, Alastair Graham² \\ From World Society of Cardiothoracic Surgeons 25th Anniversary Congress, Edinburgh \\ Edinburgh, UK. 19-22 September 2015
}

\section{Background/Introduction}

This 62 year-old gentleman presented with increasing shortness of breath on exertion and reduced exercise tolerance (NYHA class III). Brain natriuretic peptide and D-dimer were markedly elevated. ECG showed no evidence of acute ischaemia. There was a history of gastro-oesophageal reflux disease and excessive alcohol consumption 8 years previously. He was an ex-smoker with a 20 pack year history.

\section{Aims/Objectives}

CT-PA was performed to investigate for possible pulmonary embolism. A $78 \times 51 \mathrm{~mm}$ right atrial mass was identified with associated pericardial and pleural effusions. Transthoracic Echo confirmed mass was prolapsing across tricuspid valve into right ventricle. CT also showed liver cirrhosis with a large mass within segments IVa and VIII which extended into the middle hepatic vein, inferior vena cava and right atrium.

\section{Method}

After median sternotomy cardiopulmonary bypass was instituted with arterial cannula via the ascending aorta and venous cannulae via superior vena cava and right femoral vein. Cannulation of the inferior vena cava was avoided as it was obstructed by the mass. Superior vena cava and inferior vena cava were then snugged.

The procedure was performed without cardioplegia arrest (on-pump beating heart): right atrium opened; tumour inspected and delivered. The right ventricle was flushed out with saline whilst the main pulmonary artery was compressed.

\section{Results}

The delivered tumour weighed $93 \mathrm{~g}$ and measured $86 \times$ $62 \times 39 \mathrm{~mm}$. Morphological features of which are consistent with metastatic hepatocellular carcinoma. There was a significant fall in central venous pressure postoperation, from $29 \mathrm{mmHg}$ to $11 \mathrm{mmHg}$. Patient was admitted to HDU and extubated in 3 hours. He was discharged home two weeks post-operatively after having a $20 \mathrm{~kg}$ diuresis. His hepatocellular carcinoma is managed palliatively with prognosis between 3 and 6 months. He is currently being considered for biological treatment with sorafenib.

\section{Discussion/Conclusion}

Although a rare cause of an intra-cardiac mass, metastatic hepatocellular tumours can be treated similarly to renal cell carcinoma tumours of the heart. Surgical removal of the mass is an effective palliative procedure to improve quality of life. Communication with patients can be difficult as these heart tumours can be the initial presentation of metastatic tumour.

\section{Consent}

Written informed consent was obtained from the patient for publication of this abstract and any accompanying images. A copy of the written consent is available for review by the Editor of this journal

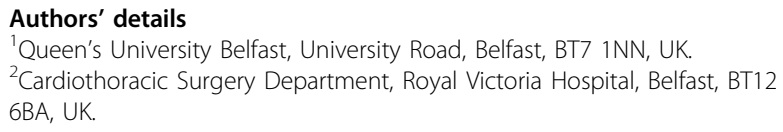

Published: 16 December 2015

doi:10.1186/1749-8090-10-S1-A80

Cite this article as: Law et al:: A rare cause of a right atrial mass. Journal of Cardiothoracic Surgery 2015 10(Suppl 1):A80. 